Pacific Journal of Mathematics

CARMAN SUBALGEBRAS OF BANACH-LIE ALGEBRAS OF 


\title{
CARTAN SUBALGEBRAS OF BANACH-LIE ALGEBRAS OF OPERATORS
}

\author{
Humberto R. Alagia
}

\begin{abstract}
A method to determine the Cartan subalgebras of certain real Banach-Lie algebras of operators is described. The algebras in question are the real, simple, separable, infinite dimensional $\mathrm{L}^{*}$-algebras, realized as algebras of HilbertSchmidt operators.
\end{abstract}

1. Introduction. Banach-Lie algebras appear in many different contexts. One particularly interesting class of examples is provided by $L^{*}$-algebras, first studied by Schue ([7], [8]). An $L^{*}$-algebra $L$ is a Lie algebra (over the real or complex field) whose underlying vector space is a Hilbert space together with a "star map" $x \mapsto x^{*}$ satisfying the condition $\langle[x, y], z\rangle=\left\langle y,\left[x^{*}, z\right]\right\rangle$ for every $x$, $y, z \in L$. Here $\langle$,$\rangle denotes the inner product in the Hilbert space$ $L$ and [,] the product in the Lie algebra $L$.

If $L$ is a finite dimensional semisimple Lie algebra over the complex field, then every compact real form $L_{0}$ of $L$ determines a structure of $L^{*}$-algebra. Indeed, if $\sigma$ denotes the conjugation of $L$ with respect to $L_{0}$ define an inner product by $\langle x, y\rangle=B(x, \sigma(y))$ for every $x, y \in L$, where $B$ is the Killing form of $L$, and set $x^{*}=-\sigma(x)$. Then it is easily checked that $\left(L,\langle\rangle,,{ }^{*}\right)$ is a complex $L^{*}$-algebra.

Infinite dimensional examples are provided by the associative $H^{*}$-algebras of Ambrose [1], considered as Lie algebras by setting $[x, y]=x y-y x$.

An $L^{*}$-algebra is semisimple if its center is $\{0\}$. This paper is concerned with infinite dimensional, separable $L^{*}$-algebras which are moreover simple (i.e., do not contain any closed ideals different from $\{0\}$ and $L)$. For all definitions and basic facts of the general theory, see [7], [8], [5].

Let $\mathscr{H}$ denote an infinite dimensional, separable complex Hilbert space. The set $L_{2}(\mathscr{H})$ of all Hilbert-Schmidt operators on $\mathscr{C}$ has a structure of $L^{*}$-algebra, where $\langle$,$\rangle is the usual inner product de-$ fined on $L_{2}(\mathscr{C})$ and the *-operation is the operation of taking the adjoint of an operator $T$. This $L^{*}$-algebra will be denoted by $L_{A}$; it is a simple $L^{*}$-algebra of type $\mathrm{A}$ and all other simple $L^{*}$-algebras are isomorphic (up to a multiple of the inner product) to a (real or complex) $L^{*}$-subalgebra of $L_{A}$. In particular $L_{B}$ and $L_{C}$ will denote the simple, complex $L^{*}$-algebras of type $\mathrm{B}$ and $\mathrm{C}$ respectively, considered as subalgebras of $L_{A}$. For details see [7], [3], [4], [10].

In the following list $\tilde{L}$ denotes a simple complex $L^{*}$-algebra $\left(L_{A}\right.$, 
$L_{B}$ or $L_{C}$ ), $\theta$ an involutive automorphism of $\widetilde{L}$ and $L$ the real form associated to $\theta$. $\theta_{U}$ is the automorphism of $\widetilde{L}$ defined by $T \rightarrow U T U^{-1}$ and $\varphi_{J}$ is the automorphism of $\widetilde{L}$ defined by $T \rightarrow-J T J^{-1}$. (Concerning the group $\operatorname{Aut}(L)$ of automorphisms of an $L^{*}$-algebra see [3], [4], [10].) All the operators act on the fixed Hilbert space $\mathscr{H}$.

\section{Type A.}

$\widetilde{L}=L_{A}=\{T: T$ is a Hilbert-Schmidt operator on $\mathscr{H}\}$.

AI : $\theta=\varphi_{J}, J$ fixed conjugation on $\mathscr{H}$

$$
L=L_{\mathrm{AI}}=\left\{T \in L_{A}: T J=J T\right\}
$$

AII : $\theta=\varphi_{J}, J$ fixed anticonjugation on $\mathscr{H}$

$$
L=L_{\mathrm{AII}}=\left\{T \in L_{A}: T J=J T\right\}
$$

AIII: $\theta=\theta_{U}, U$ unitary operator on $\mathscr{C}, U^{2}=I$,

$\mathscr{H}_{U}^{+}=\{x \in \mathscr{H}: U x=x\} \operatorname{dim} \mathscr{H}_{U}^{+}=n, n \geqq 1$ or infinite.

$$
L=L_{\mathrm{AIII}(n)}=\left\{T \in L_{A}: T^{*} U+U T=0\right\}
$$

Type B.

$J^{C}$ denotes a fixed conjugation on $\mathscr{X}$ and

$$
\widetilde{L}=L_{B}=\left\{T \in L_{A}: T^{*} J^{C}+J^{C} T=0\right\}
$$

BI : $\theta=\theta_{U}, U$ unitary operator on $\mathscr{C}, U J^{C}=J^{C} U, U^{2}=I$

$$
\operatorname{dim} \mathscr{H}_{U}^{+}=n, n \geqq 1 \text { or infinite }
$$

$$
L=L_{\mathrm{BI}(\mathfrak{n})}=\left\{T \in L_{B}: T^{*} U+U T=0\right\}
$$

BII: $\theta=\theta_{U}, U$ unitary, $U J^{c}=J^{C} U, U^{2}=-I$

$$
L=L_{\mathrm{BII}}=\left\{T \in L_{B}: T^{*} U+U T=0\right\}
$$

\section{Type C.}

$J^{a}$ denotes a fixed anticonjugation on $\mathscr{H}$

$$
\tilde{L}=L_{C}=\left\{T \in L_{A}: T^{*} J^{a}+J^{a} T=0\right\}
$$

CI : $\theta=\theta_{U}, U$ as in BII

$$
L=L_{\mathrm{CI}}=\left\{T \in L_{c}: T^{*} U+U T=0\right\}
$$

CII: $\theta=\theta_{U}, U$ as in $\mathrm{BI}$

$$
L=L_{\mathrm{CII}(\mathfrak{n})}=\left\{T \in L_{C}: T^{*} U+U T=0\right\}
$$

Now let $L$ be a semisimple $L^{*}$-algebra and $\operatorname{Aut}(L)$ its group of $L^{*}$-automorphisms. If $L$ is complex and finite dimensional a classical result states that any two Cartan subalgebras are conjugate under $\operatorname{Aut}(L)$. The result does not hold for real Lie algebras but still there is only a finite number of conjugate classes ([6], [9]). For complex, infinite dimensional, separable $L^{*}$-algebras it is shown in [2] that any two Cartan subalgebras of a simple $L^{*}$-algebra of type $\mathrm{A}$ or $\mathrm{C}$ are conjugate, whereas in type $\mathrm{B}$ there are two conjugate classes (a consequence of the fact that in infinite dimensions the classical Cartan types B and D coalesce).

This paper concerns the determination of the Cartan subalgebras 
in real simple $L^{*}$-algebras up to conjugacy under the full group of $L^{*}$-automorphisms. Using the realizations listed above, the problem reduces to the determination of Cartan subalgebras of $L^{*}$-algebras of Hilbert-Schmidt operators, which are commutative families of compact normal operators. Thus the problem is amenable to treatment by elementary operator theory techniques.

The method can be applied more generally to other Banach-Lie algebras of operators as pointed out in [5]. The reader is referred to this work as a general reference to the whole subject.

Since the actual determination of the Cartan subalgebras becomes rather tedious and repetitive, the details will be restricted mainly to algebras of type A. This will be sufficient illustration of how the method works. In the other cases, the results will simply be listed.

2. $\mathfrak{h}$-eigenvalues and automorphisms, Let $\mathfrak{h}$ be a real abelian $L^{*}$-algebra of Hilbert-Schmidt operators acting on the complex separable infinite dimensional Hilbert space $\mathscr{H}$. Let $\mathfrak{h}^{*}$ denote the space of all bounded linear functionals on the real Hilbert space $\mathfrak{y}$ and let $\left(\mathfrak{h}^{*}\right)^{c}$ denote its complexification. The elements of $\left(\mathfrak{G}^{*}\right)^{\boldsymbol{c}}$ are of the form $\mu=\alpha+i \beta$, with $\alpha, \beta \in \mathfrak{h}^{*}$. $\left(\mathfrak{h}^{*}\right)^{c}$ is the space of all bounded $\mathscr{R}$-linear maps $\mu: \mathfrak{h} \rightarrow \boldsymbol{C}$.

An element $\mu \in\left(\mathfrak{h}^{*}\right)^{c}$ is said to be an $\mathfrak{y}$-eigenvalue if the closed subspace of $\mathscr{C}$

$$
\mathscr{H}_{\mu}=\{x \in \mathscr{K}: H x=\mu(H) x \quad \forall H \in \mathfrak{h}\}
$$

is $\neq\{0\} . \quad \mathscr{C}_{\mu}$ is the corresponding $\mathfrak{h}$-eigenspace.

The existence of $\mathfrak{h}$-eigenvalues follows from the spectral theorem. In fact, $\mathfrak{h}$ is a commutative set of compact normal operators acting on $\mathscr{H}$ and thus can be simultaneously diagonalized.

Proposition 2.1. The Hilbert space $\mathscr{H}$ admits the direct sum decomposition into orthogonal h-eigenspaces $\mathscr{H}=\sum_{\mu} \mathscr{H}_{\mu}$ where $\mu$ ranges over the whole sequence of h-eigenvalues. Each subspace $\mathscr{H}_{\mu}$ is h-invariant and finite dimensional, except possibly when $\mu \equiv 0$.

Proof. If $\mu$ is an $h$-eigenvalue then $\mu(H)$ is an eigenvalue for the operator $H$, for all $H \in \mathfrak{h}$ and $\mathscr{H}_{\mu} \subset \mathscr{H}_{\mu(H)}=H$-eigenspace corresponding to $\mu(H)$. If $\lambda$ is another $h$-eigenvalue with $\mu \neq \lambda$ choose $H$ such that $\mu(H) \neq \lambda(H)$. Then $\mathscr{H}_{\mu_{(H)}}$ and $\mathscr{H}_{\lambda(H)}$ are mutually orthogonal. Thus $\mathscr{H}_{\mu}$ and $\mathscr{H}_{\lambda}$ are mutually orthogonal. Since $\mathscr{C}$ is separable, there are at most countably many h-eigenvalues. Each eigenspace is finite dimensional (except possibly if $\mu \equiv 0$ ) because 
every $H \in \mathfrak{h}$ is a compact operator.

Consider the decomposition $\mathfrak{h}=\mathfrak{h}^{+}+\mathfrak{h}^{-}$where $\mathfrak{h}^{+}=\left\{H: H^{*}=-H\right\}$ and $\mathfrak{h}^{-}=\left\{H: H^{*}=H\right\}$ the so called "*-decomposition" of $\mathfrak{h}$ as sum of its "toroidal" part h' plus its "vector part" h- (see [9]). Then for an $\mathfrak{h}$-eigenvalue $\mu, \mu(H)$ is real if $H \in \mathfrak{h}^{-}$and $\mu(H)$ is purely imaginary if $H \in \mathfrak{h}^{+}$.

An h-eigenvalue $\mu \neq 0$ is said to be real (resp. imaginary) if $\mu(\mathfrak{h}) \subset \boldsymbol{R}$ (resp. $\mu(\mathfrak{G}) \subset i \boldsymbol{R})$. If $\mu$ is neither real nor imaginary it will be called complex.

Consider an automorphism $\sigma$ of the $L^{*}$-algebra $L_{A}$ of all HilbertSchmidt operators on $\mathscr{C}$ and let $\mathfrak{h}_{1}=\sigma(\mathfrak{h})$. Then $\mathfrak{h}_{1}$ is also a real abelian $L^{*}$-subalgebra of $L_{A}$. The unitary operator $\sigma$ induces an orthogonal map (again denoted by $\sigma$ ), $\sigma: \mathfrak{h} \rightarrow \mathfrak{h}_{1}$, which in turn induces a linear map $\sigma^{*}:\left(\mathfrak{h}_{1}^{*}\right)^{c} \rightarrow\left(\mathfrak{h}^{*}\right)^{c}$ defined by $\sigma^{*}\left(\mu^{\prime}\right)=\mu^{\prime} \circ \sigma$, for $\mu^{\prime} \in\left(\mathfrak{h}_{1}^{*}\right)^{c}$. It is easily verified that $\sigma^{*}$ is a norm-preserving isomorphism.

It is known that the automorphisms $\sigma$ of $L_{A}$ can be of one of the following types:

(1) $\sigma=\theta_{U}: T \longrightarrow U T U^{-1}$,

(2) $\sigma=\varphi_{J}: T \longrightarrow-J T J^{-1}$

where $U$ is a unitary operator on $\mathscr{C}$ and $J$ a conjugate unitary operator (see [3], [4], [10]).

For $H \in \mathfrak{h}$ let $H^{\prime}=\sigma(H) \in \mathfrak{K}_{1}$ and for an $\mathfrak{h}_{1}$-eigenvalue $\mu^{\prime}$, let $\mu=\sigma^{*}\left(\mu^{\prime}\right)$.

First assume (1) holds; then $H^{\prime}=U H U^{-1}$ and if $y \in \mathscr{X}_{\mu^{\prime}}, H U^{-1} y=$ $U^{-1} H^{\prime} y=U^{-1} \mu^{\prime}\left(H^{\prime}\right) y=\mu^{\prime}\left(H^{\prime}\right) U^{-1} y=\mu^{\prime} \circ \sigma(H) U^{-1} y=\sigma^{*}\left(\mu^{\prime}\right)(H) U^{-1} y$ i.e., $H\left(U^{-1} y\right)=\mu(H) U^{-1} y$. Hence $\mu$ is an h-eigenvalue and $\mathscr{H}_{\mu}=$ $U^{-1} \mathscr{H}_{\mu}$. If (2) holds a similar argument shows that $H J^{-1} y=-$ $\mu(H) J^{-1} y$. Thus, $-\mu$ is an h-eigenvalue and $\mathscr{C}_{\mu}=J^{-1} \mathscr{H}_{\mu}$.

To unify the notation, $\sigma^{*}$ will denote either $\sigma^{*}$ or $-\sigma^{*}$ according to whether $\sigma=\theta_{U}$ or $\varphi_{J}$ respectively and $\mu^{\sigma}=\sigma^{*}(\mu)$ for an $\mathfrak{b}_{1}$-eigenvalue $\mu$. Thus one has the following

Proposition 2.2. Let $\mathfrak{h}$ be a real abelian $L^{*}$-subalgebra of $L_{A}$ and let $\sigma \in \operatorname{Aut}\left(L_{A}\right)$. Then the map $\mu \mapsto \mu^{\sigma}$ establishes a one-to-one correspondence between $\sigma(\mathfrak{G})$-eigenvalues and h-eigenvalues. If $\mathscr{H}_{\mu}$ denotes the $\sigma(\mathfrak{h})$-eigenspace corresponding to $\mu$ then $\mathscr{H}_{\mu^{\sigma}}=U^{-1} \mathscr{H}_{\mu}$ or $J^{-1} \mathscr{H}_{\mu}$ according to whether $\sigma=\theta_{U}$ or $\varphi_{J}$, respectively. Moreover $\mu$ is real (imaginary, complex) if and only if $\mu^{\sigma}$ is real (imaginary, complex).

Given $\mathfrak{h}$ as above, let $k$ (resp. $p, s$ ) denote the number of real (resp. imaginary, complex) h-eigenvalues. Then $\mathfrak{h}$ is said to be of type $(k, p, s)$. One has $0 \leqq k, p, s \leqq \infty$ and since $\mathscr{H}$ is infinite dimen- 
sional at least one of $k, s$ or $p$ is $\infty$.

COROLLARY 2.3. Let $\mathfrak{h}$ be a real abelian $L_{*}$-subalgebra of $L_{A}$ and let $\sigma \in \operatorname{Aut}\left(L_{A}\right)$. Then $\mathfrak{h}$ and $\mathfrak{h}_{1}=\sigma(\mathfrak{h})$ are both of the same type. In other words, the type of $\mathfrak{h}$ is an invariant of conjugation under the group $\operatorname{Aut}\left(L_{A}\right)$.

3. Cartan subalgebras of real $L_{*}$-algebras of type A. Let $J$ denote a fixed conjugation on $\mathscr{H}$ and let $L=\left\{T \in L_{A}: T J=J T\right\}$. The elements of $L$ are called $J$-real operators. If $\mathscr{H}_{0}=\{x \in \mathscr{H}: J x=x\}$ then $\mathscr{H}_{0}$ is a real Hilbert space and $\mathscr{H}=\mathscr{H}_{0}+i \mathscr{H}_{0}$. An orthonormal basis for $\mathscr{H}_{0}$ is also an orthonormal basis for $\mathscr{H}$. With respect to such a basis, $L$ can be realized as the set of all HilbertSchmidt matrices with real entries. This is a real form of type AI.

Let $\mathfrak{h}$ be a Cartan subalgebra of $L$. The problem is to determine the conjugate class of $\mathfrak{h}$ under the group $\operatorname{Aut}(L)$. Let $\mu$ be an h-eigenvalue and let $x \in \mathscr{H}_{\mu}, x \neq 0$. Then

$$
H J x=J H x=J \mu(H) x=\overline{\mu(H)} J x \quad \forall H \in \mathfrak{h} .
$$

Since $J x \neq 0$ it follows that $\bar{\mu}$, defined by $\bar{\mu}(H)=\overline{\mu(H)}$, is an hy-eigenvalue and that $\mathscr{H}_{\bar{\mu}}=J \mathscr{H}_{\mu}$.

Next notice that $\mu \equiv 0$ is not an h-eigenvalue. Indeed, let Ker $\mathfrak{h}=\{x \in \mathscr{H}: H x=0 \forall H \in \mathfrak{h}\} ;$ this a closed subspace of $\mathscr{H}$ which is $\mathfrak{h}$ and $J$-invariant. If $\operatorname{Ker} \mathfrak{h} \neq\{0\}$ consider the orthogonal decomposition $\mathscr{H}=\operatorname{Ker} \mathfrak{h}+(\operatorname{Ker} \mathfrak{h})^{\perp}$. Any $J$-real operator $T \neq 0$ and vanishing on $(\operatorname{Ker} \mathfrak{h})^{\perp}$ commutes with $\mathfrak{h}$ and does not belong to $\mathfrak{h}$. Since $\mathfrak{h}$ is maximal abelian, this is a contradiction. For each $\mathfrak{h}$-eigenvalue $\mu$ set $\mathscr{R}_{\mu}=\mathscr{H}_{\mu}+\mathscr{H}_{\bar{\mu}}$. If $\mu \neq \bar{\mu}$, this is an orthogonal decomposition. If $\mu=\bar{\mu}, \mu$ is a real eigenvalue and $\mathscr{R}_{\mu}=\mathscr{H}_{\mu}$. It follows that $\mathscr{R}_{\mu}$ is a closed subspace of $\mathscr{H}$, invariant under $\mathfrak{h}$ and $J$. In particular

$$
\mathscr{R}_{\mu}=\mathscr{R}_{\mu}^{0}+i \mathscr{R}_{\mu}^{0}
$$

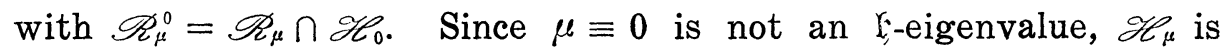
finite dimensional. Hence $\operatorname{dim}_{R} \mathscr{R}_{\mu}^{0}=\operatorname{dim}_{c} \mathscr{R}_{\mu}$ is finite for all $\mu$. $\mathscr{R}_{\mu}$ is even dimensional except when $\mu$ is real. Furthermore if $T$ is any $J$-real operator and $x \in \mathscr{H}_{0}, J T x=T J x=T x$. In particular $\mathscr{R}_{\mu}^{0}$ is h-invariant. Combining these observations with Proposition 2.1, one obtains an orthogonal decomposition of the real Hilbert space $\mathscr{H}_{0}$,

$$
\mathscr{L}_{0}=\sum_{\mu} \mathscr{R}_{\mu}^{0}
$$


into $\mathfrak{k}$-invariant finite dimensional subspaces, where $\mu$ ranges over the whole sequence of $\mathfrak{h}$-eigenvalues.

If $\mu$ is a real eigenvalue then any $H \in \mathfrak{h}^{+}$(i.e., $H$ skew-symmetric) vanishes on $\mathscr{R}_{\mu}$ and hence on $\mathscr{R}_{\mu}^{0}$. It follows that the restriction of any $H \in \mathfrak{h}$ to $\mathscr{R}_{\mu}^{0}$ is a scalar matrix $\mu(H) I$ (relative to any basis). Assume that $\operatorname{dim} \mathscr{R}_{\mu}^{0}>1$; then any operator $\neq 0$ en $\mathscr{C}_{0}$ which is diagonal on $\mathscr{R}_{\mu}^{0}$, but not a multiple of the identity, and $\equiv 0$ on $\left(\mathscr{R}_{\mu}^{0}\right)^{\perp}$ commutes with $\mathfrak{h}$ but does not belong to $\mathfrak{h}$. This contradicts the maximality of $\mathfrak{h}$. Therefore, if $\mu$ is real, $\operatorname{dim} \mathscr{R}_{\mu}=1$.

If $\mu$ is complex (neither real nor imaginary), consider the restriction of $\mathfrak{h}$ to the finite dimensional Hilbert space $\mathscr{R}_{\mu}^{0}$. This is a commutative set of normal operators; hence there is an orthonormal basis of $\mathscr{R}_{\mu}^{0}$ relative to which the matrices of $H \in \mathfrak{h}$, restricted to $\mathscr{R}_{\mu}^{0}$ consist of $1 / 2 \mathrm{dim} \mathscr{R}_{\mu}^{0}$ diagonal blocks of the form

$$
\left[\begin{array}{rr}
h & a \\
-a & h
\end{array}\right]
$$

where $\mathfrak{h}=\mu\left(H^{-}\right) \in \boldsymbol{R}, a= \pm i \mu\left(H^{+}\right) \in \boldsymbol{R} . \quad$ A maximality argument as above shows that there is only one block. Hence, $\operatorname{dim} \mathscr{R}_{\mu}^{0}=2$, when $\mu$ is complex.

Finally, if $\mu$ is imaginary, $\mathfrak{h}$ acts on $\mathscr{R}_{\mu}^{0}$ as a commutative set of skew-symmetric operators. Again there is an orthonormal basis relative to which the restrictions of $H \in \mathfrak{h}$ to $\mathscr{R}_{\mu}^{0}$ consist of diagonal blocks as above but with $h=0, a= \pm \mu(H) \in \boldsymbol{R}$. But this situation is imposible since any operator $\equiv 0$ on $\left(\mathscr{R}_{\mu}^{0}\right)^{\perp}$, with a matrix like the above on $\mathscr{R}_{\mu}^{0}$ but with nonzero diagonal, would commute with $\mathfrak{h}$. Hence, there are no imaginary h-eigenvalues.

Let $\lambda$ range over all real eigenvalues and $\mu$ over all complex ones. Then the decomposition (3.1) can be rewritten:

$$
\mathscr{H}_{0}=\sum_{\mu} \mathscr{R}_{\mu}^{0}+\sum_{\lambda} \cdot \mathscr{R}_{\lambda}^{0}
$$

with $\operatorname{dim} \mathscr{R}_{\mu}^{0}=2, \operatorname{dim} \mathscr{R}_{\lambda}^{0}=1$.

The type of $h$ (see $\S 2$ ) is $(k, 0, s)$ with $0 \leqq k, s \leqq \infty$ and at least one is $\infty$.

Construct an orthonormal basis of $\mathscr{H}_{0}$ by taking a unit vector in each $\mathscr{R}_{\lambda}{ }^{0}, \lambda$ real, and an orthonormal basis of $\mathscr{R}_{\mu}{ }^{0}$, for each complex $\mu$ such that each $H \in \mathfrak{h}$ restricted to this subspace has the form (3.2). This is also an orthonormal basis of $\mathscr{H}$ and by means of this basis $\mathfrak{h}$ is realized as an algebra of Hilbert-Schmidt matrices. The form of the matrices is determined by the pair $(k, s)$ according to the following possible cases:

(a) $0 \leqq s<\infty, k=\infty$. By suitably ordering the eigenvalues, write 


$$
\mathscr{H}_{0}=\sum_{j=1}^{s} \mathscr{R}_{\mu_{j}}^{0}+\sum_{j=2 s+1}^{\infty} \mathscr{R}_{i_{j}}^{0}
$$

and construct an ordered orthonormal basis as described above. Then $h$ consists of matrices with $s$ diagonal blocks of the form (3.2) and an infinite diagonal $\left(h_{2 s+1}, \cdots \cdots\right)$. The maximality of $\mathfrak{b}$ implies that $b$ consists of all such matrices with real entries.

(b) $0 \leqq k<\infty, s=\infty$. In this case, construct the basis according to

$$
\mathscr{C}_{0}=\sum_{j=1}^{k} \cdot \mathscr{R}_{\lambda_{j}}^{0}+\sum_{j=k+1}^{\infty} \mathscr{R}_{\lambda_{j}}^{n} \cdot
$$

Hence $\mathfrak{h}$ consists exactly of all matrices with real entries, beginning with a finite diagonal $\left(h_{1}, \cdots, h_{l_{k}}\right)$ followed by infinitely many diagonal blocks of the form (3.2).

(c) $k=\infty, s=\infty$. Put

$$
\mathscr{H}_{0}=\sum_{j=1}^{\infty} \cdot \mathscr{R}_{\ell^{\prime} j}^{0}+\sum_{j=1}^{\infty} \cdot \mathscr{R}_{\lambda_{j}}^{0}
$$

and obtain a basis relative to which consists of all real matrices with two diagonal infinite blocks, the first consisting of infinitely many diagonal blocks of the form (3.2), the second an infinite diagonal matrix.

Each of the matrix realizations obtained depends only on the type of $\mathfrak{h}$. Such a matrix realization will be called a standard matrix realization of $\mathfrak{h}$ and the associated orthonormal basis of $\mathscr{Y}_{0}$ will be called compatible with $\mathfrak{h}$.

THEOREM 3.1. Two Cartan subalgebras $\mathfrak{h}$ and $\mathfrak{h}^{\prime}$ of $L$ are conjugate under the group $\operatorname{Aut}(L)$ if and only if they are of the same type.

Proof. Assume $\mathfrak{h}$ and $\mathfrak{h}^{\prime}$ are of the same type. Let $\Phi$ and $\Phi^{\prime}$ be bases of $\mathscr{H}_{0}$ compatible with $\mathfrak{h}$ and $\mathfrak{h}^{\prime}$ respectively. Then the corresponding matrix realizations are identical. Let $U$ be the unitary operator of $\mathscr{P}$ defined by $U \varphi_{j}=\varphi_{j}^{\prime}, \varphi_{j} \in \Phi, \varphi_{j}^{\prime} \in \Phi^{\prime}$. Since $U$ leaves $\mathscr{L}_{0}$ invariant, $U J=J U$. Therefore the automorphism of $L_{A}, \theta_{U}: T \rightarrow U T U^{-1}$ leaves $L$ invariant. Thus, its restriction to $L$, again denoted by $\theta_{U}$, is an automorphism of $L$. Obviously $\theta_{U}(\mathfrak{h})=\mathfrak{h}^{\prime}$.

Conversely, let $\sigma \in \operatorname{Aut}(L)$ be such that $\mathfrak{h}^{\prime}=\sigma(\mathfrak{h})$. Extend $\sigma$ to an automorphism of $L_{A}$, again denoted by $\sigma$. It follows by Corollary 2.3 that $\mathfrak{h}$ and $\mathfrak{h}^{\prime}$ are of the same type.

If $\mathfrak{h}$ is of type $(\infty, 0, s)$ it follows from case (a) above that $\operatorname{dim} \mathfrak{h}^{+}=s$. Hence 
CoRollary 3.2. Let $\mathfrak{h}$ and $\mathfrak{h}$ be two Cartan subalgebras of $L$ satisfying $\operatorname{dim} \mathfrak{h}^{+}=\operatorname{dim} \mathfrak{h}^{+}<\infty$. Then $\mathfrak{h}$ and $\mathfrak{h}^{\prime}$ are conjugate under $\operatorname{Aut}(L)$.

The real forms of type AII can be dealt with in a similar fashion. It turns out that any Cartan subalgebra is of type $(0,0$, $\infty)$. Theorem 3.1 is valid in this case and hence any two Cartan subalgebras are conjugate under $\operatorname{Aut}(L)$. This result also holds for finite dimensional real simple Lie algebras of type AII ([9]).

The real forms of type AIII present some interesting new features. Let $U$ be a unitary involution of $\mathscr{H}$, i.e., $U$ is a unitary operator with $U^{2}=I$. Then $\mathscr{C}$ admits the orthogonal decomposition

$$
\mathscr{H}=\mathscr{H}_{U}^{+}+\mathscr{H}_{U}^{-}
$$

with $\mathscr{K}_{U}^{ \pm}=\{x \in \mathscr{K}: U x= \pm x\}$. The involutive automorphism $\theta_{U}$ of $L_{A}$ gives rise to the real form

$$
L_{U}=L=\left\{T \in L_{A}: T^{*} U+U T=0\right\}
$$

of type AIII. If $V$ is another unitary involution of $\mathscr{C}$, then $L_{t r}$ and $L_{V}$ are conjugate under $\operatorname{Aut}\left(L_{A}\right)$ if and only if either

(i ) $\operatorname{dim} \mathscr{K}_{U}^{+}=\operatorname{dim} \mathscr{X}_{V}^{+}$and $\operatorname{dim} \mathscr{H}_{U}^{-}=\operatorname{dim} \mathscr{H}_{V}^{-}$

(ii) $\operatorname{dim} \mathscr{H}_{U}^{+}=\operatorname{dim} \mathscr{H}_{V}^{-}$and $\operatorname{dim} \mathscr{H}_{U}^{-}=\operatorname{dim} \mathscr{H}_{V}^{+}$, (see [3], [4], [10]).

Since $L_{U}=L_{-U}$ it may be assumed without loss of generality that $\operatorname{dim} \mathscr{H}_{U}^{+}=\infty$. Hence $L_{U^{\top}}$ and $L_{V}$ are conjugate if and only if $\operatorname{dim} \mathscr{H}_{U}^{-}=\operatorname{dim} \mathscr{H}_{V}^{-}$.

For each $n \geqq 0$ or $n=\infty$ let $U$ be such that $\operatorname{dim} \mathscr{H}_{U}^{-}=n$; then $\left\{L_{A I I(n)}=L_{U}: n \geqq 0\right.$ or $\left.\infty\right\}$ gives a complete set of representatives of conjugate classes of real forms of type AIII. (If $n=0, U=I$ and $L_{U}$ is the "compact" real form of $L_{A}$. We shall assume $U \neq I$.)

Let $\mathfrak{h}$ be a Cartan subalgebra of $L=L_{U}$ and let $\mu$ be an h-eigenvalue. Since $H U=-U H^{*}$ for all $H \in \mathfrak{h}$, one has

$$
H U x=-U H^{*} x=-U \mu\left(H^{*}\right) x=-\mu\left(H^{*}\right) U x, \quad \forall x \in \mathscr{H}_{\mu} .
$$

This shows that $\mu$ defined by $\tilde{\mu}(H)=-\mu\left(H^{*}\right)$, is also an $\mathfrak{h}$-eigenvalue and $\mathscr{H}_{\tilde{\mu}}=U \mathscr{H}_{\mu}$.

Since for every $\mu, \mu(H)=\overline{\mu\left(H^{*}\right)}$ one has $\tilde{\mu}(H)=-\overline{\mu(H)}$, i.e. $\tilde{\mu}=-\bar{\mu}$. In particular $\mu=\tilde{\mu}$ if and only if $\mu$ is imaginary. Thus,

$$
\mathscr{R}_{\mu}=\mathscr{H}_{\mu}+\mathscr{H}_{\mu}
$$

is a closed subspace of $\mathscr{H}$-invariant under $\mathfrak{h}$ and $U$. This is an 
orthogonal decomposition, unless $\mu$ is imaginary in which case $\mathscr{R}_{\mu}=$ $\mathscr{H}_{\mu}=\mathscr{H}_{\tilde{\mu}}$.

Since $\mathscr{R}_{\mu}$ is $U$-invariant, there is an orthogonal decomposition

$$
\mathscr{R}_{\mu}=\mathscr{R}_{\mu}^{+}+\mathscr{R}_{\mu}^{-}
$$

where

$$
\mathscr{R}_{\mu}^{ \pm}=\mathscr{R}_{\mu} \cap \mathscr{H}_{\mu}^{ \pm}
$$

First notice that an argument similar to that used in case AI shows that $\mu \equiv 0$ is not an h-eigenvalue.

If the eigenvalue $\mu$ is not imaginary there exists $H \in \mathfrak{h}^{-}$such that $\mu(H) \neq 0$. In this case $H: \mathscr{R}_{\mu}^{+} \rightarrow \mathscr{R}_{\mu}^{-}$is an isomorphism. Indeed, any self-adjoint $T \in L$ maps $\mathscr{H}_{U}^{+}$into $\mathscr{H}_{U}^{-}$; it follows that $H \in \mathfrak{h}^{-}$maps $\mathscr{R}_{\mu}{ }^{+}$into $\mathscr{R}_{\mu}{ }^{-}$. This maps is $1: 1$. Let $v \in \mathscr{R}_{\mu}{ }^{+}$and write $v=x+\widetilde{x}$ according to (3.8). Then $H v=\mu(H) x+\tilde{\mu}(H) \widetilde{x}=\mu(H)(x-\widetilde{x})$. Hence $H v=0$ if and only if $x-\widetilde{x}=0$, i.e., if and only if $v=0$.

To see that $H$ is onto let $w \in \mathscr{R}_{\mu}^{-}$and write $w=y+\widetilde{y}$, according to (3.8). Set $v=(1 / \mu(H))(y-\widetilde{y})$, then $v \in \mathscr{R}_{\mu}$ and $H v=w$. One can easily check that $U v=v$ and so $v \in \mathscr{R}_{\mu}{ }^{+}$.

Therefore $\operatorname{dim} \mathscr{R}_{\mu}=2 \operatorname{dim} \mathscr{R}_{\mu}{ }^{+}=2 \operatorname{dim} \mathscr{R}_{\mu}^{-}$, is even if $\mu$ is not imaginary.

Using that $\mathscr{H}_{\tilde{\mu}}=U \mathscr{H}_{\mu}$, choose an orthonormal basis of $\mathscr{R}_{\mu}$, $\Phi_{\mu}=\left\{\varphi_{j}: 1 \leqq j \leqq 2 q\right\}, 2 q=\operatorname{dim} \mathscr{R}_{\mu}$, such that $\varphi_{q+j}=U \varphi_{j} 1 \leqq j \leqq q$.

If $H \in \mathfrak{h}^{+}$, then $H \varphi_{j}=\mu(H) \varphi_{j}, 1 \leqq j \leqq 2 q$. On the other hand if $H \in \mathfrak{h}^{-}$,

$$
H \varphi_{j}=\mu(H) \varphi_{j} ; \quad H \varphi_{q+j}=-\mu(H) \varphi_{q+j}, \quad 1 \leqq j \leqq q .
$$

Define a new orthonormal basis of $\mathscr{R}_{\mu}, \Psi_{\mu}=\left\{\psi_{j}: 1 \leqq j \leqq 2 q\right\}$ by

$$
\psi_{2 k-1}=\frac{1}{\sqrt{2}}\left(\varphi_{k}+\sqrt{-1} \varphi_{q+k}\right) ; \quad \psi_{2 k}=\frac{1}{\sqrt{2}}\left(\sqrt{-1} \varphi_{k}+\varphi_{q+k}\right),
$$

$1 \leqq k \leqq q$

An easy computation shows that, if $H \in \mathfrak{h}^{+}$

$$
H \psi_{j}=\mu(H) \psi_{j}, \quad 1 \leqq j \leqq 2 q
$$

and that if $H \in \mathfrak{h}^{-}$

$$
H \psi_{2 k-1}=-\sqrt{-1} \mu(H) \psi_{2 k} ; \quad H \psi_{2 k}=\sqrt{-1} \mu(H) \psi_{2 k-1}
$$

for $1 \leqq k \leqq q$.

Moreover

$$
U \psi_{2 k-1}=\psi_{2 k} ; \quad U \psi_{2 k}=\psi_{2 k-1} \quad 1 \leqq k \leqq q .
$$

Therefore relative to $\Psi_{\mu}$ the matrices of the restrictions of $H$ 
to $\mathscr{R}_{\mu}$ consist of $q$ diagonal blocks of the form

$$
\left[\begin{array}{rr}
h & a \\
-a & h
\end{array}\right]
$$

with $h=\mu\left(H^{+}\right), a=i \mu\left(H^{-}\right)$, whereas the matrix of $U$ consists of $q$ diagonal blocks of the form

$$
\left[\begin{array}{ll}
0 & 1 \\
1 & 0
\end{array}\right]
$$

Then the maximality of $\mathfrak{h}$ implies that $q=1$, i.e., $\operatorname{dim} \mathscr{R}_{\mu}=2$. Remember that this is valid under the assumption that $\mu$ is not imaginary. Using this one concludes easily that there are no real eigenvalues.

If $\mu$ is imaginary, then $\mathscr{R}_{\mu}=\mathscr{H}_{\mu}=\mathscr{H}_{\tilde{\mu}}$ and it can be easily shown that $\operatorname{dim} \mathscr{R}_{\mu}=1$.

Summing up, there is an orthogonal decomposition

$$
\mathscr{H}=\sum_{\mu} \mathscr{R}_{\mu}
$$

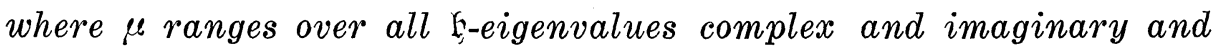
where the subspaces $\mathscr{R}_{\mu}$ are $\mathfrak{h}$ and U-invariant and $\operatorname{dim} \mathscr{R}_{\mu}=2$ if $\mu$ is complex, $\operatorname{dim} \mathscr{R}_{\mu}=1$ if $\mu$ is imaginary.

Assume now that $\operatorname{dim} \mathscr{H}_{U}^{-}=n<\infty$. Since $\mathscr{R}_{U}-\perp \mathscr{R}_{\lambda}-$ if the eigenvalues $\mu, \lambda$ are different and since $\mathscr{R}_{\mu}^{-} \subset \mathscr{H}_{U}^{-}$one has: $\sum_{\mu} \operatorname{dim} \mathscr{R}_{\mu}^{-}=$ $\operatorname{dim} \mathscr{H}_{U}^{-}=n$.

By the preceding considerations, if $\mu$ is complex then $\operatorname{dim} \mathscr{R}_{\mu}^{-}=$ $1 / 2 \operatorname{dim} \mathscr{R}_{\mu}=1$. Thus the number $s$ of complex eigenvalues is $\leqq n$ and any Cartan subalgebra is of type $(0, \infty, s)$ where $s \leqq n$. The number $q=n-s$ is the number of imaginary eigenvalues $\nu$ such that $\mathscr{R}_{\nu}^{-}=\mathscr{R}_{\nu} \subset \mathscr{H}_{U}^{-}$.

Proceeding as in the case AI one can construct a "compatible basis for $\mathfrak{h}^{\prime \prime}$ of the Hilbert space $\mathscr{H}$ relative to which $\mathfrak{h}$ consists of all matrices in $L$ beginning with $s$ diagonal blocks of the form (3.9) followed by an infinite diagonal $\left(h_{s+1}, \cdots\right)$, all entries being imaginary numbers. Relative to the same basis the matrix of $U$ consists of diagonal blocks of the form (3.10) followed by an infinite diagonal $(-1, \cdots,-1,+1,+1, \cdots)$ where the number of $(-1)$ 's is $n-s$.

THEOREM 3.3. Two Cartan subalgebras $\mathfrak{h}$ and $\mathfrak{h}^{\prime}$ of a real form of type $\operatorname{AIII}(n), 0<n<\infty$, are conjugate under $\operatorname{Aut}(L)$ if and only if they are of the same type. 
Proof. The "only if" part follows from Corollary 2.3. Conversely assume that $\mathfrak{h}$ and $\mathfrak{h}^{\prime}$ are of the same type. Let $\Phi$ and $\Phi^{\prime}$ be orthonormal bases compatible with $\mathfrak{h}$ and $\mathfrak{h}^{\prime}$ respectively. Let $V$ be the unitary operator of $\mathscr{H}$ mapping $\Phi$ onto $\Phi^{\prime}$. The corresponding automorphism $\theta_{V}$ of $L_{A}$ maps $\mathfrak{h}$ onto $\mathfrak{h}^{\prime}$.

It remains to check that $\theta_{V}$ leaves the real form $L$ invariant. This is an immediate consequence of the fact that the matrices of $U$ relative to $\Phi$ and $\Phi^{\prime}$ are identical.

It follows that the conjugate class of $\mathfrak{h}$ is completely determined by $\operatorname{dim} \mathfrak{h}^{-}=s \leqq n$.

COROLlary 3.4. Two Cartan subalgebras of a real form $L$ of type $\operatorname{AIII}(n), 0<n<\infty$, are conjugate if and only if $\operatorname{dim} \mathfrak{h}^{-}=$ $\operatorname{dim} \mathfrak{h}^{\prime} \leqq n$. There are exactly $(n+1)$ conjugate classes.

4. Cartan subalgebras of real forms of type $\operatorname{AIII}(\infty)$. Unlike the real forms $\operatorname{AIII}(n)$, the conjugate classes of Cartan subalgebras in the case $\operatorname{AIII}(\infty)$ are not completely determined by their type. The difficulty arises from the fact that the (-1)-eigenspace of the defining unitary operator is infinite dimensional.

In this case $\operatorname{dim} \mathscr{H}_{U}^{-}=\operatorname{dim} \mathscr{H}_{U}^{+}=\infty$. Proceeding as in the case $n<\infty$, one obtains an orthonormal basis of $\mathscr{H}$ and a coresponding standard matrix realization of a Cartan subalgebra $\mathfrak{h}$. This realization is completely determined by the type of $\mathfrak{h}$. But in this case the number of complex eigenvalues can be arbitrary, finite or infinite.

If $\mathfrak{h}$ is of type $(0, \infty, s), s<\infty$, the matrix of $U$ relative to $\Phi$ has necessarily infinitely many -1 in the diagonal. Thus it is completely determined by the type of $\mathfrak{h}$. However, if $\mathfrak{h}$ is of type $(0$, $p, \infty)$, with $p$ finite, the matrix of $U$ relative to $\Phi$ has as many -1 in the diagonal as subspaces $\mathscr{R}_{\nu}$ are contained in $\mathscr{H}_{U}^{-}(\nu$ imaginary eigenvalue).

To handle this case consider the orthogonal decomposition. $\mathscr{H}=\sum_{\nu} \mathscr{R}_{\nu}+\sum_{\mu} \mathscr{R}_{\mu}$, where $\nu$ (resp. $\mu$ ) ranges over all imaginary (resp. complex) h-eigenvalues. One has $\operatorname{dim} \mathscr{R}_{\mu}{ }^{+}=1=\operatorname{dim} \mathscr{R}_{\nu}^{-}$, $\operatorname{dim} \mathscr{R}_{\nu}=1$ and $\mathscr{R}_{2} \subset \mathscr{H}_{U}^{ \pm}$. Call an imaginary eigenvalue $\nu$ positive (resp. negative) if $\mathscr{R}_{\nu} \subset \mathscr{H}_{U}^{+}$(resp. $\mathscr{R}_{\nu} \subset \mathscr{H}_{U}^{-}$) and write $\nu>0$ (resp. $\nu<0)$.

If $\mathfrak{h}$ is of type $(0, p, s)$, then

$$
p=p^{+}+p^{-}
$$

where $p^{+}$(resp. $p^{-}$) is the number of positive (resp. negative) imaginary eigenvalues. These three numbers $p, p^{+}, p^{-}$can be arbit- 
rary, finite or infinite.

Let $\mathscr{H}_{1}$ be the closed subspace of $\mathscr{C}$ defined by

$$
\mathscr{H}_{1}=\sum_{\nu} \cdot \mathscr{R}_{\nu}=\sum_{\nu>0} \cdot \mathscr{R}_{\nu}+\sum_{\nu<0} \cdot \mathscr{R}_{\nu} \cdot
$$

$\mathscr{H}_{1}$ is invariant under $\mathfrak{h}$ and $U$; set $U_{1}=\left.U\right|_{\pi_{1}}$.

Let $\mathfrak{h}^{\prime}$ be another Cartan subalgebra of $L$ of type $\left(0, p^{\prime}, s^{\prime}\right)$. Denote by $\alpha$ (resp. $\beta$ ) the imaginary (resp. complex) h'-eigenvalues. Then as in (4.1),

$$
p^{\prime}=p^{++}+p^{\prime-}
$$

and set

$$
\mathscr{H}_{2}=\sum_{\alpha} \cdot \mathscr{R}_{\alpha}=\sum_{\alpha>0} \mathscr{R}_{\alpha}+\sum_{\alpha<0} \cdot \mathscr{R}_{\alpha} .
$$

$\mathscr{K}_{2}$ is invariant under 5 and $U$; set $U_{2}=\left.U\right|_{\mathscr{C}_{2}}$.

LEMMA 4.1. The notations being as above assume that $\mathfrak{h}$ and $\mathfrak{h}^{\prime}$ are of the same type $(0, p, s)$. Let $p=p^{+}+p^{-}=p^{++}+p^{\prime-}$. If one of the following two conditions is satisfied

(a) $p^{+}=p^{++}$and $p^{-}=p^{\prime-}$

(b) $p^{+}=p^{\prime-}$ and $p^{-}=p^{p^{+}}$

then $\mathfrak{h}$ and $\mathfrak{b}^{\prime}$ are conjugate under $\operatorname{Aut}(L)$.

Proof. Let $\Phi$ and $\Phi^{\prime}$ be orthonormal basis of $\mathscr{C}$ compatible with $\mathfrak{h}$ and $\mathfrak{h}^{\prime}$ respectively. If (a) holds then the matrices of $U$ relative to $\Phi$ and $\Phi^{\prime}$ are identical. Then the proof of Theorem 3.3 is valid in this case and $\mathfrak{b}$ is conjugate to $\mathfrak{h}^{\prime}$.

If (b) holds, let $W$ be an involutive unitary operator on $\mathscr{C}$ satisfying $W U=-U W$. Then $W$ maps $\mathscr{H}_{U}{ }^{+}$onto $\mathscr{H}_{U}^{-}$and $\mathscr{H}_{U}^{-}$onto $\mathscr{H}_{U}^{+}$. (The operator $W$ can be constructed since $\operatorname{dim} \mathscr{H}_{U}^{+}=\operatorname{dim} \mathscr{K}_{U}^{-}$.) It follows that the automorphism $\theta_{W}$ of $L_{A}$ leaves $L$ invariant. Thus $\zeta_{1}=\theta_{V V}(\hat{i})$ is a Cartan subalgebra of $L$ of type $(0, p, s)$. The condition $W U=-U W$ implies that $p^{+}=p_{1}^{-}$and $p^{-}=p_{1}^{+} . \quad \mathrm{By}(\mathrm{b}), p^{\prime+}=p_{1}^{+}$ and $p^{\prime-}=p_{1}^{-}$and, by the first part of the proof, $\mathfrak{h}^{\prime}$ and $\mathfrak{h}_{1}$ are conjugate. Therefore $\mathfrak{h}$ is conjugate to $\mathfrak{h}^{\prime}$.

Let $\mathfrak{b}$ be a Cartan subalgebra of $L$ of type $(0, p, s)$. The signature of $h$ is the (unordered) pair $\left\{p^{+}, p^{-}\right\}$. Notice that if $p$ is finite, the signature of $\mathfrak{h}$ is the signature of the Hermitian form $U_{1}$ on the finite dimensional Hilbert space $\mathscr{H}_{1}$.

Lemma 4.1 shows that if $\mathfrak{h}$ and $\mathfrak{h}^{\prime}$ are of the same type and have the same signature they are conjugate. This condition is also necessary, as shown below. 
Let $\sigma$ be an automorphism of $L_{A}$ such that

(1) $\sigma(\mathfrak{h})=\mathfrak{h}^{\prime}$;

(2) $\sigma$ leaves $L$ invariant.

By Corollary $2.3 \mathfrak{h}$ and $\mathfrak{h}^{\prime}$ are of the same type $(0, p, s)$.

Assume that $\sigma=\theta_{V}, V$ an unitary operator on $\mathscr{H}$. The following arguments also hold with minor modifications if $\sigma=\varphi_{J}, J$ a conjugate unitary operator.

According to Proposition 2.2 each imaginary $\mathfrak{h}^{\prime}$-eigenvalue $\alpha$ is

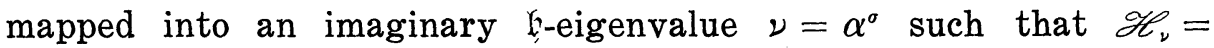
$V^{-1}\left(\mathscr{H}_{\alpha}\right)$. Therefore $\mathscr{R}_{\alpha}=V\left(\mathscr{R}_{\nu}\right)$ and the operator $V$ maps the subspace $\mathscr{H}_{1}$ onto $\mathscr{H}_{2}((4.3),(4.4))$. In particular $\mathscr{H}_{1}$ and $\mathscr{H}_{2}$ have the same dimension $p \leqq \infty$. For $i=1,2$ set

$$
L_{i}=\left\{T \in L_{2}\left(\mathscr{H}_{i}\right): T^{*} U_{i}+U_{i} T=0\right\}
$$

where $L_{2}\left(\mathscr{H}_{i}\right)$ is the set of all Hilbert-Schmidt operators on $\mathscr{H}_{i}$. If $p=\infty, L_{i}$ is a real simple separable $L^{*}$-algebra of type AIII. If $p<\infty$, then

$$
\mathfrak{s} L_{i}=\left\{T \in L_{i}: \text { trace } T=0\right\}
$$

is a real simple Lie algebra isomorphic to $\mathfrak{H} \mathfrak{u}\left(p^{+}, p^{-}\right)\left(\right.$resp. $\left.\mathfrak{Z} \mathfrak{u}\left({p^{\prime}}^{+}, p^{\prime-}\right)\right)$ the Lie algebra of the special unitary group on $\mathscr{H}_{i}$ relative to the Hermitian form $U_{i}$.

Proposition 4.2. Assume that hy and $\mathfrak{h}^{\prime}$ are conjugate under $\operatorname{Aut}(L)$. Then they are of the same type and have the same signature.

Proof. The only thing to prove is that $\mathfrak{h}$ and $\mathfrak{h}^{\prime}$ have the same signature. The algebras $L_{1}, L_{2}$ defined above can be naturally identified with the subalgebras of $L$

$$
\left\{T \in L: T\left(\mathscr{H}_{i}\right) \subset \mathscr{H}_{i} \text { and } T \equiv 0 \text { on } \mathscr{H}_{i}{ }^{\perp}\right\}, \quad i=1,2 .
$$

With this identification $L_{1}$ and $L_{2}$ become $L^{*}$-subalgebras of $L$ and, if $p<\infty$, so do $: L_{1}$ and $: L_{2}$.

The automorphism $\theta_{V}$ maps $L_{1}$ isomorphically onto $L_{2}$. Indeed, assume that $T\left(\mathscr{H}_{1}\right) \subset \mathscr{H}_{1}$ and $T \equiv 0$ on $\mathscr{H}_{1}{ }^{\perp}$. Since $V$ is unitary and maps $\mathscr{H}_{1}$ onto $\mathscr{H}_{2}$ it follows that $S=V T V^{-1}$ leaves $\mathscr{H}_{2}$ invariant and vanishes on $\mathscr{H}_{2}{ }^{\perp}$.

Thus if $p=\infty, L_{1}$ and $L_{2}$ are isomorphic real $L^{*}$-algebras of type AIII. By (3.7) it follows that $\mathfrak{h}$ and $\mathfrak{h}^{\prime}$ have the same signature.

On the other hand if $p<\infty, \theta_{V}$ sets up an isomorphism between $\mathfrak{s} L_{1}$ and $\mathfrak{s} L_{2}$. By a well-known result, the signatures of $U_{1}$ and $U_{2}$ 
are equal.

\section{A summary of other results.}

Type B. As remarked in the introduction, the complex $L^{*}$-algebra $L_{B}$ has two conjugate classes of Cartan subalgebras. If $\widetilde{\mathfrak{h}}$ is a Cartan subalgebra of $L_{B}$ and if $\operatorname{Ker} \widetilde{\mathfrak{h}}=\{H \in \widetilde{\mathfrak{h}}: H x=0 \forall x \in \mathscr{K} \mathcal{C}\}$ then either (I) $\operatorname{dim} \operatorname{Ker} \widetilde{\mathfrak{h}}=0$ or (II) $\operatorname{dim} \operatorname{Ker} \widetilde{\mathfrak{h}}=1$. According to whether (I) or (II) holds, $\widetilde{\mathrm{h}}$ is said to be of type I or II respectively ([2]). If $\mathfrak{h}$ is a Cartan subalgebra of a real form $L$ of $L_{B}$, let $\widetilde{\mathfrak{h}}$ denote its complexification. Then $\operatorname{Ker} \mathfrak{h}=\operatorname{Ker} \widetilde{\mathfrak{h}}$ and $\mathfrak{h}$ is said to be of type I or II according to the type of $\widetilde{\mathfrak{h}}$. It follows easily that if $\mathfrak{h}_{1}, \mathfrak{h}_{2}$ are Cartan subalgebras of $L$ such that $\widetilde{\mathfrak{h}}_{1}, \widetilde{\mathfrak{h}}_{2}$ are not conjugate under $\operatorname{Aut}\left(L_{B}\right)$, then $\mathfrak{K}_{1}, \mathfrak{K}_{2}$ are not conjugate under $\operatorname{Aut}(L)$.

If $L$ is a real form of type BI and $\mathfrak{h}$ a Cartan subalgebra then $\mu \equiv 0$ is a an $\mathfrak{h}$-eigenvalue if and only if $\mathfrak{h}$ is of type II. Accordingly $\mathfrak{h}$ can be of "type $\mathrm{I}-(k, p, s)$, or "II- $(k, p, s)$ ".

Let $U$ be the unitary involution defining the real form $L$; for each $n \geqq 1$ or infinite there is a real form determined by $\operatorname{dim} \mathscr{H}_{U}{ }^{-}=n$.

If $n$ is finite the determination of the possible h-eigenvalues leads to the conclusion that there are necessarily infinitely many imaginary eigenvalues and only a finite number of real and complex eigenvalues. One obtains a standard matrix realization of $\mathfrak{h}$ which depends only on its type $(\mathrm{I}-(k, \infty, s)$ or II $-(k, \infty, s))$. Two Cartan subalgebras are conjugate under $\operatorname{Aut}(L)$ if and only if they are of the same type.

If $n=\infty, \operatorname{dim} \mathscr{H}_{U}^{-}=\infty$ and it turns out that the values of $k, p, s$ can be arbitrary finite or infinite. This forces an analysis similar to the one in case $\operatorname{AIII}(\infty)$ which takes into account the number of imaginary eigenvalues $\nu$ such that the subspaces $\mathscr{R}_{\nu}=$ $\mathscr{H}_{\mu}+\mathscr{H}_{\mu} \subset \mathscr{H}_{U}^{-}$. The signature of a Cartan subalgebra can be similarly defined. Two Cartan subalgebras turn out to be conjugate if and only if their types and signatures are equal.

For real forms of type BII one shows that $\mu \equiv 0$ cannot be an eigenvalue for a Cartan subalgebra $\mathfrak{h}$. Moreover there are no real eigenvalues and any $\mathfrak{h}$ is of type $(0, \infty, s)$ with $s<\infty$ or $(0, p, \infty)$, $p<\infty$. Again two Cartan subalgebras are conjugate under $\operatorname{Aut}(L)$ if and only if their types are the same.

Type C. For a Cartan subalgebra of a real $L^{*}$-algebra of type $\mathrm{C}, \mu \equiv 0$ cannot be an eigenvalue.

If $L$ is of type CI, then a Cartan subalgebra $\mathfrak{h}$ is of type $(k$, 
$p, s)$ where the numbers $k, p, s$ can be arbitrary finite or infinite (subject to the condition that at least one of them be $\infty$ ). The conjugate class of $\mathfrak{h}$ is completely determined by its type.

If $L$ is of type CII then one must distinguish two cases according to whether $\operatorname{dim} \mathscr{H}_{U}^{-}=n<\infty$ or $\operatorname{dim} \mathscr{H}_{U}^{-}=\infty$.

In either case there are no real h-eigenvalues.

If $n<\infty$, the conjugate class of $\mathfrak{h}$ is completely determined by its type. If $n=\infty$ one proceeds analogously to cases $\operatorname{AIII}(\infty)$ and $\mathrm{BI}(\infty)$ defining the signature of $\mathfrak{h}$. The type and signature constitute a complete set of invariants of the Cartan subalgebra.

\section{REFERENCES}

1. W. Ambrose, Structure Theorems for a special class of Banach algebras, Trans. Amer. Math. Soc., 57 (1945), 364-386.

2. V. K. Balachandran, Simple $L^{*}$-algebras of classical type, Math. Ann., 180 (1969), 163-170.

3. - Real $L^{*}$-algebras, preprint.

4. P. de la Harpe, Classification des $L^{*}$-algèbres semi-simples réelles séparables, C. R. Acad. Sci. Paris, Sér. A, 272 (1971), 1559-1561.

5. - Classical Banach-Lie algebras and Banach-Lie groups of operators in Hilbert space, Lecture Notes in Math $\mathrm{N}^{\circ} 285$, Springer-Verlag, 1972.

6. B. Kostant, On the conjugacy of real Cartan subalgebras, I, Proc. Nat. Acad. Sci. U.S.A., 41 (1955), 967-970.

7. J. R. Schue, Hilbert space methods in the theory of Lie algebras, Trans. Amer. Math. Soc., 95 (1960), 69-80.

8. - Cartan decompositions for $L^{*}$-algebras, Trans. Amer. Math. Soc., 98 (1961), 334-349.

9. M. Sugiura, Conjugate classes of Cartan subalgebras in real semisimple Lie algebras, J. Math. Soc. Japan, 11 (1959), 374-434.

10. I. Unsain, Classification of the simple separable real $L^{*}$-algebras, J. Differential Geometry, 7 (1972), 423-451.

Received July 9, 1980. Partially supported by SECYT (Argentina) grants 8903/79 and 9091/80-16.

IMAF, Universidad Nacional de Córdoba

5000 Cordoba, ARgentina 



\title{
PACIFIC JOURNAL OF MATHEMATICS
}

\section{EDITORS}

\author{
DONALD BABBITT (Managing Editor) \\ University of California \\ Los Angeles, CA 90024 \\ Hugo RossI \\ University of Utah \\ Salt Lake City, UT 84112 \\ C. C. Moore and ANDrew OGG \\ University of California \\ Berkeley, CA 94720
}

\section{J. DugundjI}

Department of Mathematics

University of Southern California

Los Angeles, CA 90007

R. FinN and J. Milgram

Stanford University

Stanford, CA 94305

\section{ASSOCIATE EDITORS}
R. ARENS
E. F. BeCKENBACH
B. H. NeUMANN
F. WOLF
K. YoshidA

\section{SUPPORTING INSTITUTIONS}

UNIVERSITY OF ARIZONA

UNIVERSITY OF BRITISH COLUMBIA

CALIFORNIA INSTITUTE OF TECHNOLOGY

UNIVERSITY OF CALIFORNIA

MONTANA STATE UNIVERSITY

UNIVERSITY OF NEVADA, RENO

NEW MEXICO STATE UNIVERSITY

OREGON STATE UNIVERSITY

\author{
UNIVERSITY OF OREGON \\ UNIVERSITY OF SOUTHERN CALIFORNIA \\ STANFORD UNIVERSITY \\ UNIVERSITY OF HAWAII \\ UNIVERSITY OF TOKYO \\ UNIVERSITY OF UTAH \\ WASHINGTON STATE UNIVERSITY \\ UNIVERSITY OF WASHINGTON
}

The Supporting Institutions listed above contribute to the cost of publication of this Journal, but they are not owners or publishers and have no responsibility for its content or policies.

Mathematical papers intended for publication in the Pacific Journal of Mathematics should be in typed form or offset-reproduced, (not dittoed), double spaced with large margins. Please do not use built up fractions in the text of the manuscript. However, you may use them in the displayed equations. Underline Greek letters in red, German in green, and script in blue. The first paragraph or two must be capable of being used separately as a synopsis of the entire paper. Please propose a heading for the odd numbered pages of less than 35 characters. Manuscripts, in triplicate, may be sent to any one of the editors. Please classify according to the scheme of Math. Reviews, Index to Vol. 39. Supply name and address of author to whom proofs should be sent. All other communications should be addressed to the managing editor, or Elaine Barth, University of California, Los Angeles, California, 90024.

50 reprints to each author are provided free for each article, only if page charges have been substantially paid. Additional copies may be obtained at cost in multiples of 50 .

The Pacific Journal of Mathematics is issued monthly as of January 1966. Regular subscription rate: $\$ 102.00$ a year (6 Vols., 12 issues). Special rate: $\$ 51.00$ a year to individual members of supporting institutions.

Subscriptions, orders for numbers issued in the last three calendar years, and changes of address shoud be sent to Pacific Journal of Mathematics, P.O. Box 969, Carmel Valley, CA 93924, U.S.A. Old back numbers obtainable from Kraus Periodicals Co., Route 100, Millwood, NY 10546.

PUBLISHED BY PACIFIC JOURNAL OF MATHEMATICS, A NON-PROFIT CORPORATION

Printed at Kokusai Bunken Insatsusha (International Academic Printing Co., Ltd.). 8-8, 3-chome, Takadanobaba, Shinjuku-ku, Tokyo 160, Japan. 


\section{Pacific Journal of Mathematics}

\section{Vol. 98, No. 1 \\ March, 1982}

Humberto Raul Alagia, Cartan subalgebras of Banach-Lie algebras of

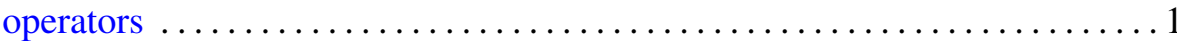

Tom M. (Mike) Apostol and Thiennu H. Vu, Elementary proofs of

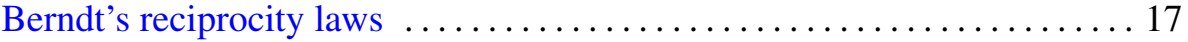

James Robert Boone, A note on linearly ordered net spaces $\ldots \ldots \ldots \ldots . \ldots 25$

Miriam Cohen, A Morita context related to finite automorphism groups of



Willibald Doeringer, Exceptional values of differential polynomials . . . . . . 55

Alan Stewart Dow and Ortwin Joachim Martin Forster, Absolute

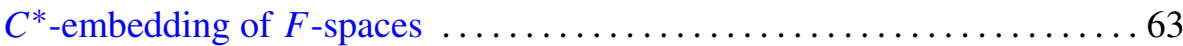

Patrick Hudson Flinn, A characterization of $M$-ideals in $B\left(l_{p}\right)$ for

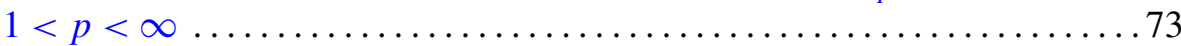

Jack Emile Girolo, Approximating compact sets in normed linear spaces . . . 81

Antonio Granata, A geometric characterization of $n$th order convex



Kenneth Richard Johnson, A reciprocity law for Ramanujan sums . . . . . . .99

Grigori Abramovich Kolesnik, On the order of $\zeta\left(\frac{1}{2}+i t\right)$ and $\Delta(R) \ldots \ldots 107$

Daniel Joseph Madden and William Yslas Vélez, Polynomials that

represent quadratic residues at primitive roots $\ldots \ldots \ldots \ldots \ldots \ldots \ldots 123$

Ernest A. Michael, On maps related to $\sigma$-locally finite and $\sigma$-discrete

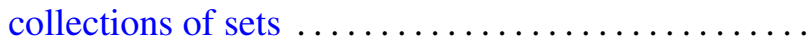

Jean-Pierre Rosay, Un exemple d'ouvert borné de $\mathbf{C}^{3}$ “taut" mais non

hyperbolique complet

Roger Sherwood Schlafly, Universal connections: the local problem

Russel A. Smucker, Quasidiagonal weighted shifts .....

Eduardo Daniel Sontag, Remarks on piecewise-linear algebra . .

Jan Søreng, Symmetric shift registers. II

H. M. (Hari Mohan) Srivastava, Some biorthogonal polynomials suggested

by the Laguerre polynomials 